\title{
Aluminum Potassium Sulfate and Tannic Acid Injection for Hemorrhoids
}

\author{
Seok Won Lim \\ Hang Clinic of Coloproctology, Seoul, Korea
}

A quick hemostatic effect, as well as sclerosing and shrinkage of hemorrhoids, can be attained when internal hemorrhoids are treated by using injection therapy with aluminum potassium sulfate and tannic acid (ALTA), the outcomes of treatment may be similar to those of a hemorrhoidectomy. However, if the type of hemorrhoid or the method of injection is not appropriate for ALTA treatment, complications peculiar to ALTA or recurrence may develop. Accordingly, sufficient understanding of the treatment mechanism of ALTA injection and repeated training for injection are required for effective use of the ALTA treatment.

Keywords: Aluminum potassium sulfate and tannic acid, Hemorrhoid, Injection therapy

\section{INTRODUCTION}

In the past, $5 \%$ phenol almond oil (PAO) was most widely used for sclerotherapy for hemorrhoids [1]. The treatment mechanism of PAO injection is that PAO administered into the submucosal layer of a hemorrhoid and the hemorrhoidal pedicle induces inflammation in the areas surrounding the blood vessels in the hemorrhoids and the fibrosis resulting from the inflammation reduces blood flow into hemorrhoid and fixes the hemorrhoid to the mucosa. Accordingly, effect of PAO injection on internal hemorrhoids with bleeding last for about 1 year and, thus, is not permanent. In addition, because positive treatment outcomes are not expected for prolapsed hemorrhoids, PAO injection is not applicable to internal hemorrhoids of grade 3 to 4 [1]. However, aluminum potassium sulfate and tannic acid (ALTA) is a scientifically-improved ingredient of Xiaozhiling that has been used in China as

Received: March 2, 2012 Revised: April 12, 2012

Accepted: April 13, 2012

Correspondence to: Seok Won Lim, M.D.

Department of Surgery, Hang Clinic of Coloproctology, Kwang-an Building

\# 1643, Chowon-dong, Gwanak-gu, Seoul 151-902, Korea

Tel: +82-2-855-7175, Fax: +82-2-855-7353

E-mail: hangclinic@yahoo.co.kr

(C) 2012 The Korean Society of Coloproctology

This is an open-access article distributed under the terms of the Creative Commons Attribution NonCommercial License (http://creativecommons.org/licenses/by-nc/3.0) which permits unrestricted non-

commercial use, distribution, and reproduction in any medium, provided the original work is properly cited. an injection therapy for treating hemorrhoids and is recognized to produce positive outcomes even for grade 3 to 4 internal hemorrhoids [2-5]. Because ALTA injection has much more significant effects on inflammatory responses and fibrosis than PAO injection, ALTA injection is considered to have more lasting effects than PAO injection and to be effective for the treatment of grade 3 to 4 hemorrhoids.

Sclerotherapy of internal hemorrhoids by using ALTA is conducted through a 4-step injection, which results in treatment outcomes similar to those of a radical hemorrhoidectomy [4-7]. Because ALTA injection is effective for internal hemorrhoids only, not for external hemorrhoids, a method combining a hemorrhoidectomy and ALTA injection has been introduced recently. This hybrid method is expected to reduce postoperative pain, complications such as postoperative anal stricture [8], and delayed wound healing. In this paper, the history, pharmacological action, applications and limitations, method of administration, complications, treatment outcomes, and combined modality therapy of ALTA injection, which is a new sclerotherapy for hemorrhoids, are described.

\section{HISTORY OF ALTA}

In 1971, Shi et al. [2] developed Xiaozhiling, an upgraded version of Myongbanek, which has been traditionally used for treating hemorrhoids in China. The major ingredients of Xiaozhiling include ALTA [2]. Clinical use of Xiaozhiling was approved by the 
Chinese government in 1979; it was then introduced to Korea and Japan. The composition of ALTA was finalized after some of the additives of Xiaozhiling were modified in Japan. Experimental treatment started in 1998, and the Japanese government approved commercial use of ALTA in 2005. In Korea, ALTA is imported from Japanese pharmaceutical companies and has been used since 2007.

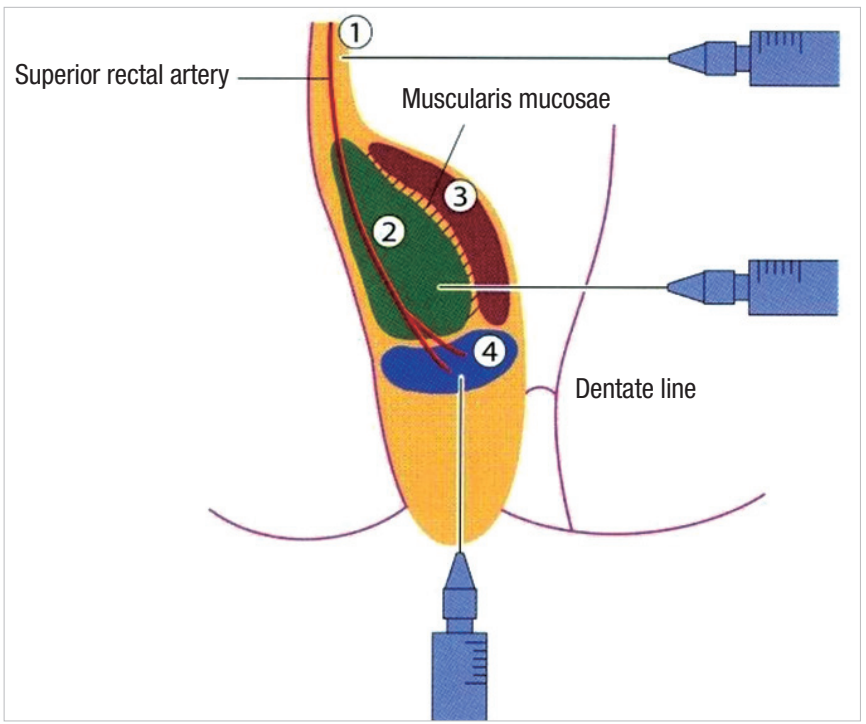

Fig. 1. The mechanism of action of aluminum potassium sulfate and tannic acid injection. (1) Submucosa at the superior pole of hemorrhoid. (2) Submucosa in the central part of hemorrhoid. (3) Mucous lamina propria in the central part of hemorrhoid. (4) Submucosa at the inferior pole of hemorrhoid.

\section{MECHANISM OF ALTA INJECTION}

Once ALTA is injected into hemorrhoids, blood flow to the hemorrhoids is interrupted, and a quick hemostatic effect and shrinkage of the hemorrhoids develop. With time, persistent fibrosis develops due to sterile inflammation; then, adhesion and fixation of the mucosa and the submucosal layer to the muscular layer is promoted. Finally, bleeding and prolapse of the hemorrhoid disappear [7] (Fig. 1).

\section{INDICATIONS OF ALTA INJECTION}

ALTA injection is effective only for internal hemorrhoids. In the case of mixed hemorrhoids having features of external piles, the application of ALTA is not appropriate. In addition, ALTA is applicable neither in cases of hemorrhoids in an acute stage, such as external thrombosed hemorrhoids and strangulated hemorrhoids, nor in cases accompanied by fibrosis and complications, such as fissures, hemorrhoidal fistulae and anal polyps. Further, in cases of large hemorrhoids that require more than $40 \mathrm{~mL}$ of ALTA administration, surgical treatments should be considered because radical treatment outcomes through ALTA injection are hardly expected.

\section{METHODS OF ALTA INJECTION}

Observance of the 4-step injection is required. The 4-step injection is a procedure of administrating appropriate amounts of ALTA into 4 parts of the hemorrhoids: the upper, shallow middle, deep middle and lower parts [4,5] (Fig. 2). At step 1, about $3 \mathrm{~mL}$ of ALTA are injected into the submucosal layer of the upper part of

\section{The mechanism of action of ALTA injection}

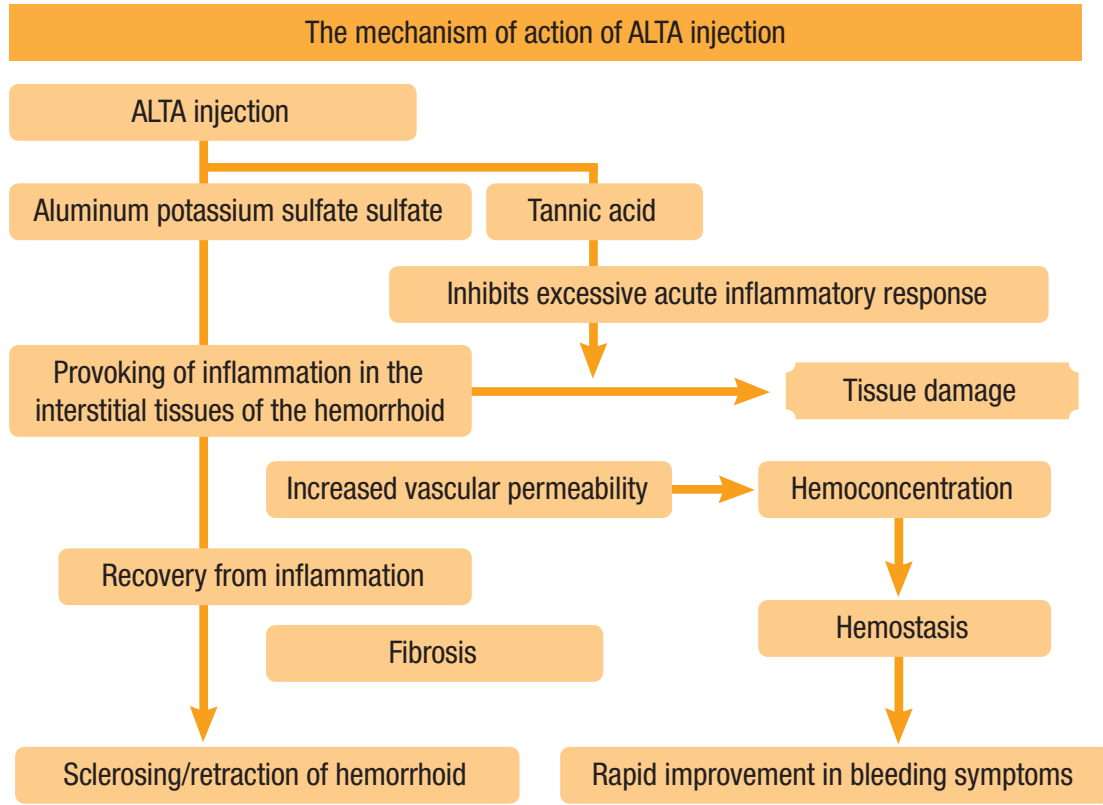

Fig. 2. Four-step injection of aluminum potassium sulfate and tannic acid (ALTA). 
the hemorrhoid. At step 2, ALTA is injected in an amount of $1 \mathrm{~mL}$ more than the volume of the hemorrhoid into the submucous layer of the middle part of the hemorrhoid. At step 3, about 1 to $2 \mathrm{~mL}$ of ALTA, which is about half the amount injected in step 2, are injected when the needle passes the lamina propria while retreating from the muscularis mucosae, the point the needle reached at step 2. At step 4, about 2 to $3 \mathrm{~mL}$ of ALTA are injected at the deep submucosal layer 0.1 to $0.2 \mathrm{~cm}$ adoral to the dentate line of the lower part of the hemorrhoid; then, an additional $1 \mathrm{~mL}$ is injected while withdrawing the needle. In cases of hemorrhoids with volumes of $1 \mathrm{~cm}^{3}$ or less and accessory hemorrhoids, only steps 2 and 3 are required. In all steps, ALTA should not be injected into the muscular layer. Use of an ALTA-only needle is advantageous because resistance from the muscular layer is easily detected, and injection into the muscular layer can be prevented. In addition, when a Ztype ALTA-only anoscope, not an ordinary anoscope, is used, hemorrhoids can be observed in front, the upper areas of the hemorrhoids can be observed, and application of fixation or compression to the injection area is easy, so the ALTA solution does not leak from the dentate line.

Upon completion of ALTA injection, massage is done over the entire injected area by using the fingers in order to diffuse the ALTA solution evenly into all areas of the hemorrhoids. Regarding the timing of the massage, a one-time massage after finishing the injection for all hemorrhoids was recommended in the past, but nowadays, immediate massage is recommended upon completion of the 4 steps for every hemorrhoid in order to prevent complications such as rectal ulcers caused by local stagnation of the ALTA solution in the hemorrhoid [9].

\section{COMPLICATIONS OF ALTA}

Complications that may develop after ALTA injection are shown in Table 1. Causes of complications may include inappropriate injection site, inappropriate dose, and excessive local stagnation. Therefore, strict observance of the 4-step injection process is required. An accurate injection site and dose should be selected, and immediate massage after ALTA is necessary $[9,10]$. Additional compli- cations may include low abdominal pain, bradycardia and hypotension after ALTA injection, which are considered to be caused by a vagovagal nerve stimulation reflex in the pelvic cavity. Therefore, use of ALTA solution mixed with lidocaine anesthetic agent may prevent symptoms of vagovagal nerve stimulation reflex from developing [6].

\section{PERFORMANCE OF ALTA INJECTION}

As a result of a multi-center study conducted by 10 institutions in Japan on prolapsed internal hemorrhoid cases in order to compare the performance of ALTA injection therapy with that of a hemorrhoidectomy, the disappearance rates at postoperative 28 days for the ALTA injection therapy group and the hemorrhoidectomy group were $94 \%$ (75/80 cases) and $99 \%$ (84/85 cases), respectively, showing no significant difference from each other, in the case of grade 3 to 4 hemorrhoids. At postoperative 1 year, the recurrence rate for the ALTA injection therapy group was 16\% (12/73 cases), which was slightly higher than the $2 \%$ (2/81 cases) for the surgery group. Considering that ALTA injection is a less invasive procedure with less pain compared with surgery and that the injection technique had not been fully mastered by the surgeons during the early periods of its use, the results obtained with ALTA treatment are considered satisfactory [5].

A study conducted by Kunimoto et al. [11] of 16 recurrent cases after ALTA injection found that the recurrence rate was high when ALTA injection therapy was first introduced because a sufficient amount of ALTA could not be injected due to the development of symptoms such as bradycardia, pain in the lower abdomen and hypotension during ALTA injection. Since then, use of a mixture of ALTA solution and lidocaine was introduced to prevent symptoms of vagovagal nerve stimulation reflex; consequently, a sufficient amount of ALTA injection was administered, and the recurrence rate decreased accordingly. According to a study on recurrence after ALTA injection, cases of internal-pile recurrence were treated with repeated ALTA injection whereas cases of external-pile recurrence were treated with a hemorrhoidectomy. When ALTA injection is performed for hemorrhoids with components of ex-

Table 1. Complications of aluminum potassium sulfate and tannic acid (ALTA) injection

\begin{tabular}{ll}
\hline Complication & \\
\hline Hard indruation & Excessive dose administration \\
& Uneven distribution of ALTA solution due to insufficient massage \\
Rectal ulcer, hemorrhoid necrosis & Injection too shallow or excessive dose administration \\
& Uneven distribution of ALTA solution due to insufficient massage \\
Rectal stricture & Excessive dose administration over 3 mL into the upper area of the hemorrhoids in step 1 \\
& Mistakenly administered dose of step 1 with that of step 2 \\
Anal pain & Injection or infiltration of ALTA solution into the dentate line, anal canal or skin \\
Rectal necrosis & Insertion of needle up to the muscular layer, resulting in excessive dose administration \\
Prostatitis, epididymitis, testitis & Penetration of needle into all layers of the prostate \\
\hline
\end{tabular}


Journal of The Korean Society of Aluminum Potassium Sulfate and Tannic Acid Injection for Hemorrhoids

Coloproctology Seok Won Lim

ternal piles, the components of the external piles still remain after treatment. Therefore, screening of inappropriate cases should be conducted in order to select cases of internal piles for ALTA injection and to reduce the recurrence rate [11].

\section{COMBINED MODALITY THERAPY OF \\ A HEMORRHOIDECTOMY AND ALTA \\ INJECTION}

ALTA injection is effective only for internal hemorrhoids. How-
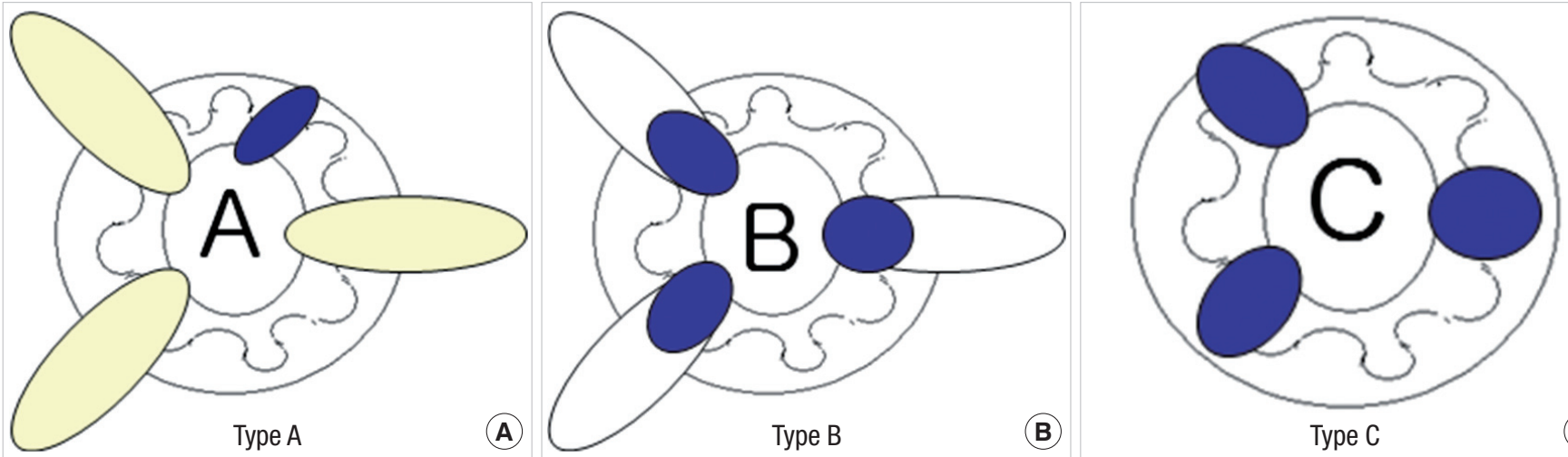

Fig. 3. Classification of aluminum potassium sulfate and tannic acid injection method. (A) Type A, accessory file; (B) Type B, big sized file; (C) Type C, complete injection.
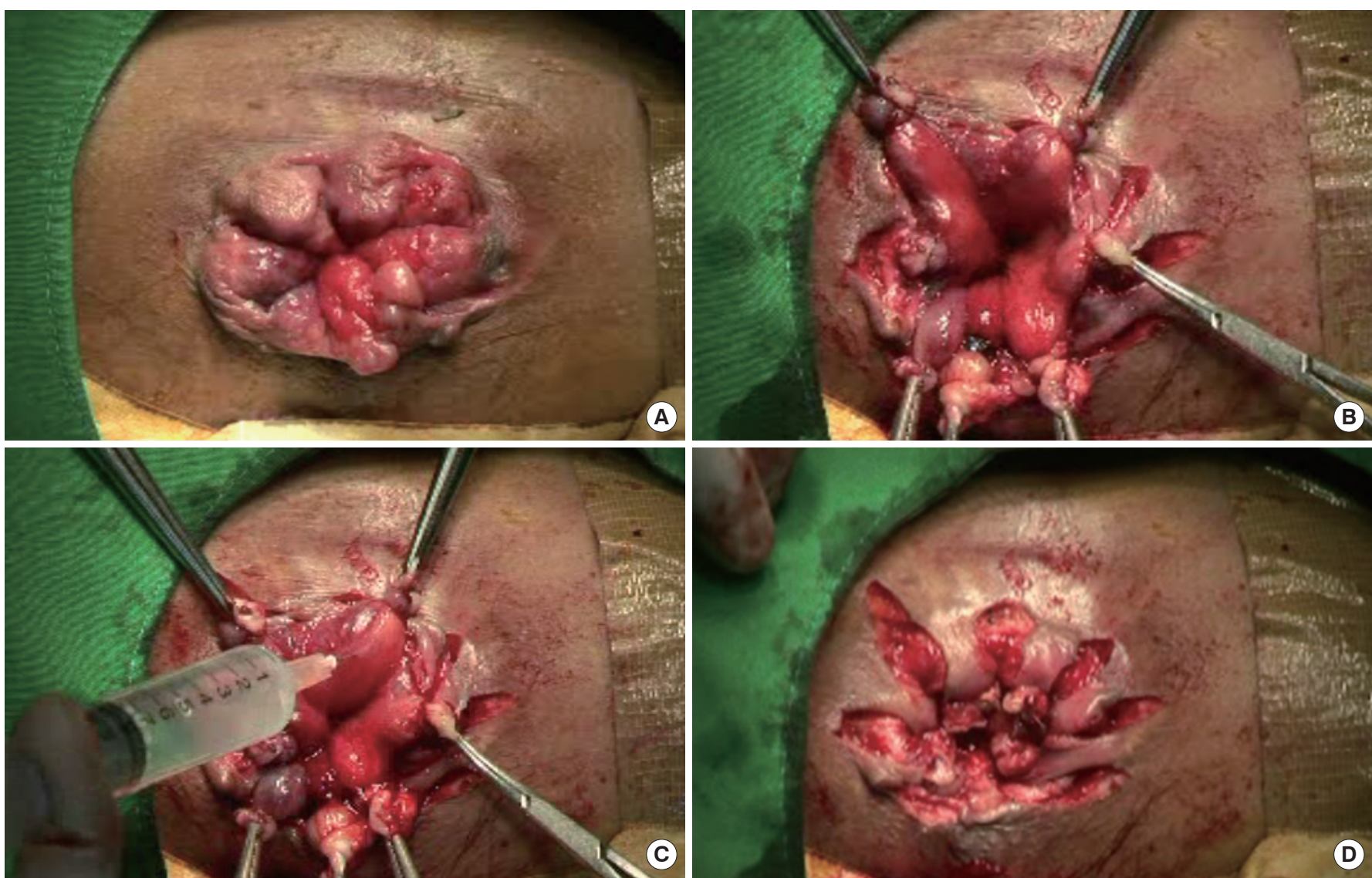

Fig. 4. Low ligation with aluminum potassium sulfate and tannic acid (ALTA) injection technique (type B, big sized file). (A) Preoperative finding. (B) Low ligation and excision. (C) ALTA injection on internal hemorrhoids. (D) Postoperative finding. 
ever, even though most mixed hemorrhoids are practically not internal piles but include external piles, if a combined modality therapy of a hemorrhoidectomy and ALTA injection is applied to mixed hemorrhoids, the volume of excision can be less than that of surgery alone; thus, the development of pain and complications such as anal stricture can be reduced, and the hospital stay and the wound healing time can be shortened, helping patients to have a faster return to normal life.

We named the ALTA injection combined modality therapy as the "hybrid hemorrhoidectomy," which has three types of injection methods (Fig. 3). Type A was named after the accessory pile. The main pile of the hemorrhoids is removed by surgery; then, ALTA injection is performed on the internal hemorrhoids included in the remaining accessory pile. Type B was named after big-sized piles. The external pile of mixed hemorrhoids is removed by surgery; then, ALTA injection is performed on the remaining internal hemorrhoids. Type $\mathrm{C}$ was named after complete injection. ALTA injection is performed for hemorrhoids composed of internal hemorrhoids only. In particular, type B is a low ligation with ALTA injection ligating and excising external piles at the dentate line and with ALTA injection into the areas of the internal piles (Fig. 4). Because the extent of excision in low ligation with ALTA injection is less than that in a conventional hemorrhoidectomy, anal stricture can be prevented when multiple piles are removed from circumferential hemorrhoids, and postoperative secondary bleeding rarely occurs due to the site of ligation and excision being close to the dentate line [8].

\section{CONCLUSION}

When ALTA injection is applied to cases of internal piles only, the outcomes of treatment are almost the same as those of a hemorrhoidectomy, but with almost no pain. In cases of mixed hemorrhoids containing external piles, a combined modality therapy of a hemorrhoidectomy and ALTA injection can be used. Compared with the results obtained when using only a hemorrhoidectomy, those obtained when using hybrid therapy of a hemorrhoidectomy and ALTA injection include less postoperative pain, fewer complications such as anal stricture, a shortened period of wound healing and a faster return to normal life. Thus, ALTA injection may be used with less pain for the radical treatment of hemorrhoids.

\section{CONFLICT OF INTEREST}

No potential conflict of interest relevant to this article was reported.

\section{REFERENCES}

1. Lim SW, Lee KR, Yoo CJ, Park SY, Kim HS, Lee JK. The effect of sclerotherapy on bleeding hemorrhoids. J Korean Soc Coloproctol 1995;11:211-6.

2. Shi Z, Zhou J, He X. On treatment of third degree internal hemorrhoids with "Xiaozhiling” injection. J Tradit Chin Med 1981;1: 115-20.

3. Shi Z. Treatment of hemorrhoid of III, IV stage by four steps injection of xiaozhiling. Zhongguo Zhong Xi Yi Jie He Za Zhi 1998; 18:201-3.

4. Takamura $H$, Inatsugu $H$, Yoshikawa S, Masuda T. Treatment of internal hemorrhoids with Xiaozhiling injection. J Jpn Soc Coloproctol 2001;54:910-4.

5. Takano M, Iwadare J, Ohba H, Takamura H, Masuda Y, Matsuo K, et al. Sclerosing therapy of internal hemorrhoids with a novel sclerosing agent. Comparison with ligation and excision. Int J Colorectal Dis 2006;21:44-51.

6. Hachiro Y, Kunimoto M, Abe T, Kusano M. A new sclerosing therapy for internal hemorrhoids: experience in 200 cases with Zione injection. J Jpn Soc Coloproctol 2006;59:317-21.

7. Ono T, Goto K, Takagi S, Iwasaki S, Komatsu H. Sclerosing effect of OC-108, a novel agent for hemorrhoids, is associated with granulomatous inflammation induced by aluminum. J Pharmacol Sci 2005;99:353-63.

8. Lim SW. Analysis and measures for anal stricture following a hemorrhoidectomy. J Korea Soc Coloproctol 2006;22:293-7.

9. Lee YC, Shin HK, Lim CH, Yang HK, Kang JH, Lee KY, et al. Comparison of Early Clinical Outcomes Between ALTA (Aluminum Potassium Sulfate and Tannic Acid, Ziohn(R)) Injection Therapy and a Submucosal Hemorrhoidectomy in Patients with Internal Hemorrhoids. J Korean Soc Coloproctol 2010;26:179-85.

10. Kunimoto M, Abe T, Hachiro Y, Tsuruma T. Treatment of rectal ulcer complicating sclerosing therapy for internal hemorrhoids with Aluminum potassium sulfate tannic acid (ALTA). J Jpn Soc Coloproctol 2007;60:327-32.

11. Kunimoto M, Abe T, Hachiro Y, Tsuruma T. Profiles of Cases of Internal Hemorrhoids Retreated after Initial Treatment by Sclerosing Therapy with Aluminum Potassium Sulfate Tannic Acid (ALTA). Can ALTA be a Successful Radical Treatment for Hemorrhoids? J Jpn Soc Coloproctol 2008;61:11-5. 